apparently not justified. The need for early diagnosis and treatment has been emphasized previously (Millichap JG et al. IAMA 1962;182:125).

\title{
ANTICONVULSANT TOXICITY
}

\section{LONG-TERM VALPROATE HEMATOLOGIC SIDE EFFECTS}

Hematologic side effects in 60 patients, aged 2-29 years (mean 14 years), receiving valproate (VPA) monotherapy for $>4$ years in a long-term care facility, are reported from the Department of Pediatrics, East Carolina University School of Medicine, Greenville, North Carolina. Hematologic abnormalities, especially thrombocytopenia, $<130,000 / \mathrm{mcl}$ (12 patients) and macrocytosis (11), were demonstrated in $20(33 \%)$ patients. With VPA levels $>100 \mathrm{mcg} / \mathrm{ml}$ in 22 patients, the incidence increased to 55\%. Platelet counts were inversely related to VPA levels; thrombocytopenia was corrected when VPA dosage was reduced. Three had anemia, and 3 had leukopenia. Serum $\mathrm{B}_{12}$ levels were increased $(>1000 \mathrm{mcg} / \mathrm{ml})$ in $51(86 \%)$; folate levels were normal. Blood smears showed increased numbers of bilobed polymorphonucleic cells (Pelger-Huet-like cells), an anomaly commonly associated with VPA-induced macrocytosis. (May RB, Sunder TR. Hematologic manifestations of long-term valproate therapy. Epilepsia Nov/Dec 1993;34:1098-1101). (Reprints: Dr RB May, Craven County Health Department, PO Drawer 12610, New Bern, NC 28561).

COMMENT. The authors recommend close regular surveillance of patients receiving valproate, with continuous attention to blood counts, especially platelets and mean corpuscular volumes. The early recognition of these relatively frequent blood count anomalies may lead to reduction in VPA dosage or drug withdrawal and avoidance of major hematologic toxicity.

\section{CARBAMAZEPINE, AUDITORY ERPs, AND BCECT}

The effects of carbamazepine (CBZ) on cognitive function were evaluated by using measurements of auditory event-related potentials (ERPs) and P300 latencies in 23 patients, aged 7 to 16 years, with benign childhood epilepsy and centrotemporal spikes (BCECT), at the Department of Pediatrics, Toyama Medical and Pharmaceutical University, Toyama, Japan. As the epilepsy was controlled at the initiation of therapy, and with increasing age, the P300 latency was at first shortened. During the course of therapy with CBZ, P300 latency was prolonged, and the age-corrected P300 latency showed a significant correlation with the serum CBZ level. The dose of CBZ ranged from $10-23 \mathrm{mg} / \mathrm{kg} /$ day (mean 15.8). The latency became shorter when CBZ was discontinued. (Naganuma Y et al. Auditory event-related potentials in benign childhood epilepsy with centrotemporal spike: The effects of carbamazepine. Clin Electroencephalogr Jan 1994;25:8-12). (Reprints: Yoshihiro Naganuma MD, Department of Pediatrics, Toyama Medical and Pharmaceutical University, 2630 Sugitani, Toyama 930-01, Japan).

COMMENT. The major positive component of auditory event-related potentials, at a latency of $300 \mathrm{msec}(\mathrm{P} 300)$ for rare tones $(2000 \mathrm{~Hz})$, has been correlated with cognitive function. Abnormalities in ERPs in patients with epilepsy, and particularly prolongation of P300 latency, have been ascribed to the effects of the seizures and to antiepileptic drug therapy. Various epileptic syndromes have shown different 
degrees of abnormality in the ERPs. In this study, after a transient beneficial response, the cumulative effect of carbamazepine was associated with a chronic impairment of cognitive function, as measured by changes in auditory event-related potentials.

Studies of the effects of carbamazepine on auditory brainstem responses $(A B R)$ in 21 epileptic patients examined at the Institute of Clinical and Experimental Neurology, Thilisi, Republic of Georgia, demonstrated prolongation of ABR peak latencies and interpeak intervals. In addition, CBZ was associated with increases in peak latencies of middle-latency responses and slow cortical potentials. CBZ has suppressive influences on central auditory structures and the acoustic nerve. (Japaridze G et al. Epilepsia Nov/Dec 1993; $34: 1105-1109$ ).

\section{CARBAMAZEPINE TOXICITY WITH GENERIC SUBSTITUTION}

Two 6-year-old children with carbamazepine (CBZ) toxicity, reported from the University of Miami School of Medicine, were found to have $22 \%$ and $41 \%$ increases in serum CBZ levels after substitution of Tegretol with the generic brand, Epitol, because of insurance company policies. Adverse effects included lethargy, ataxia, slurred speech, and nystagmus. When dosage was adjusted, symptoms of toxicity resolved. (Gilman JT, Alvarez LA, Duchowny M. Carbamazepine toxicity resulting from generic substitution. Neurology Dec 1993; 43:2696-7). (Reprints: Dr Jamie Gilman, Clinical Pharmacology, Miami Children's Hospital, 6125 SW 31st Street, Miami, FL 33155).

COMMENT. Generic substitution of Tegretol has previously been associated with lowered serum levels of CBZ and seizure exacerbation. Reduced bioavailability is also reported with moisture-exposed CBZ, resulting in status epilepticus (Bell WL et al. Epilepsia Nov/Dec 1993;34:1102-4). Gilman et al have documented 2 cases of increased bioavailability with Epitol substitution, one of 4 generic carbamazepine products available in the US. In 1988, 70 million CBZ tablets were recalled because of bioinequivalence and clinical seizure exacerbation (Oles KS, Gal P. Bioequivalency revisited: Epitol versus Tegretol. Editorial. Neurology Dec 1993;43:2435-6). Factors other than generic substitution may account for significant variations in CBZ concentrations, including interlot variability, exposure of drug to excessive heat or moisture, food and drug interactions, sample timing, and patient compliance.

\section{ATTENTION DEFICIT AND COGNITIVE DISORDERS}

\section{METHYLPHENIDATE AND SLEEP PATTERNS}

The effects of methylphenidate $(0.3-0.4 \mathrm{mg} / \mathrm{kg}) \mathrm{cf}$ placebo on sleep in 10 children with ADHD are reported in a double-blind crossover study at the Bnai Zion Medical Center and the Technion-Israel Institute of Technology, Haifa, Israel. Sleep duration was significantly shorter during the drug period compared to placebo or baseline periods. The percent of quiet sleep was lower in the ADHD study group compared with controls in baseline measures, but not during methylphenidate treatment. (Tirosh E et al. Effects of methylphenidate on sleep in children with attention-deficit hyperactivity disorder. AIDC Dec 1993; 147:1313-1315). (Reprints: Dr Tirosh, Hannah Khoushy Child Development Center, Bnai Zion Medical Center, POB 4940, Haifa, Israel). 